\title{
Dynamics of nearly unstable axisymmetric liquid bridges
}

\author{
José M. Perales and José M. Vega \\ ETSI Aeronáuticos, Universidad Politécnica de Madrid, Madrid 28040, Spain
}

\begin{abstract}
The dynamics of a noncylindrical, axisymmetric, marginally unstable liquid bridge between two equal disks is analyzed in the inviscid limit. The resulting model allows for the weakly nonlinear description of both the (first stage of) breakage for unstable configurations and the (slow) dynamics for stable configurations. The analysis is made for both slender and short liquid brides. In the former range, the dynamics breaks reflection symmetry on the midplane between the supporting disks and can be described by a standard Duffing equation, while for short bridges reflection symmetry is preserved and the equation is still Duffing-like but exhibiting a quadratic nonlinearity. The asymptotic results compare well with existing experiments.
\end{abstract}

\section{INTRODUCTION}

A liquid bridge is the configuration that appears when a liquid mass is held by capillary forces between two solid supports. The configuration offers the simplest mechanical model for some complex systems that appear in many phenomena and is of industrial interest in, e.g., the crystal growth technique known as floating zone.

In the past, one can find many papers dealing with the statics of liquid bridges. A review can be found in Meseguer et $a l .{ }^{1}$ The previous works mainly focused on determining the parameter values for which the liquid bridge is either stable or unstable. The instability (breakage) for axisymmetric liquid bridges can have two very different behaviors. For slender (long) liquid bridges the configuration breaks into two drops of different volume while for shorter bridges the two drops are equal. In both cases, after the breakage a third, much smaller drop (the so-called satellite) appears in between. For even shorter liquid bridges the instability is nonaxisymmetric and leads no longer to a breakage but to a nonaxisymmetric deformation that can be recovered by simply returning the values of the parameters to their original values.

Cylindrical liquid bridges were considered in the pioneering work by Rayleigh, ${ }^{2}$ who found that these liquid bridges destabilize when their length is greater than its circumference. More recent stability analyses have focused on the effect of various, not necessarily small perturbations, such as axial gravity/acceleration, ${ }^{3}$ unequal disks diameter, ${ }^{4}$ rigid solid rotation, ${ }^{5}$ and the combined effect of the above. ${ }^{6,7}$ In all these, although the basic configurations were axisymmetric, the possible instabilities were allowed to be either axisymmetric or nonaxisymmetric.

Additional studies cover the influence of various nonaxisymmetric perturbations. ${ }^{8,9}$ The results analysis when perturbations are small are not easy and it is often required asymptotic analysis to clarify the effect of every perturba- tion. For the case of nearly cylindrical liquid bridges, the analysis was made by Vega and Perale ${ }^{10}$ and more recently, a study has been made on the influence of small perturbations in the change of stability of liquid bridges close to the stability limit. ${ }^{11}$

The theoretical predictions mentioned above have been corroborated by experiments, either on board of space laboratories (where residual gravity is much smaller) or on ground; in the latter case, both Plateau baths and in microzones ${ }^{12-14}$ have been used. A lot of works deal with linear vibrations from the theoretical, ${ }^{15-25}$ numerical, ${ }^{26-29}$ or experimenta $1^{30}$ point of view. A less understood problem is connected with dynamics near the stability limit. Available results are really scarce and either focus only on the vicinity of the point where a cylindrical liquid bridge loses stability (the Rayleigh limit, see Sanz, ${ }^{31}$ Higuera, Nicolás, and Vega, ${ }^{32}$ and Nicolás and Vega ${ }^{33}$ or are based on onedimensional approximations, such as the so-called Cosserat model. ${ }^{34-38}$

Figure 1 shows four consecutive pictures of the breakage of a liquid bridge experiment made on board Spacelab D-2 mission (1993). This particular breakage was unexpected as the configuration was rather far from the stability limit predicted by a statics analysis and one of the goals of this work is to elaborate a theoretical model to quantitatively explain this breaking process (or the oscillatory movement that will appear in case the stability limit is not reached).

The object of the present paper is to analyze the dynamics near the instability limit for noncylindrical configuration. The geometry and the various parameters involved are sketched in Fig. 2. In the following, all lengths are made dimensionless with the mean of the two almost equal disk radii $R_{0}=\left(R_{1}+R_{2}\right) / 2$. The basic parameters defining the configuration are the slenderness $\Lambda=L /\left(2 R_{0}\right)$ and the dimensionless volume $V /\left(\pi L R_{0}{ }^{2}\right)$. The analysis will be made in the vicinity of the instability limit, which corresponds to a nondimensional volume $V_{0}$ that depends on $\Lambda$; small pertur- 

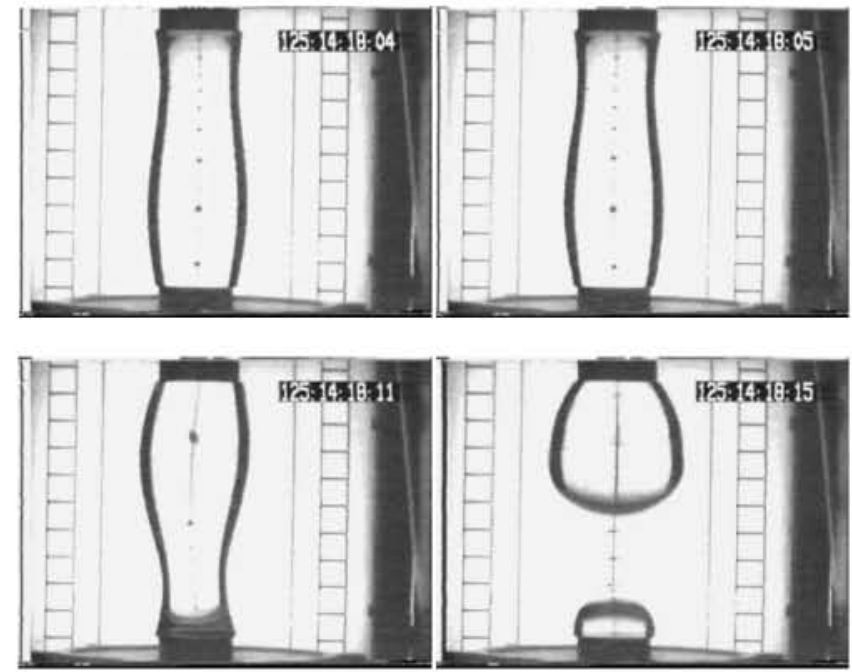

FIG. 1. Consecutive pictures of a liquid bridge breaking process. WL-AFPM-STACO experiment. Spacelab D-2 mission (1993).

bations of the nondimensional volume $V=V /\left(\pi L R_{0}{ }^{2}\right)-V_{0}$ will be considered, as well as small values of the dimensionless disk radii difference $H=\left(R_{2}-R_{1}\right) /\left(R_{2}+R_{1}\right)$ and small values of the Bond number $B=\rho g R_{0}{ }^{2} / \sigma$. Namely, we assume that

$$
\Lambda \sim 1, \quad V_{0} \sim 1, \quad|V| \ll 1, \quad|H| \ll 1, \quad|B| \ll 1 .
$$

\section{PROBLEM FORMULATION, STEADY STATES, AND INSTABILITY LIMITS}

In the following, all expressions are dimensionless, using capillary time $\left(\rho R_{0}{ }^{3} / \sigma\right)^{1 / 2}$ and the mean of the disk radii $R_{0}$ to nondimensionalize time and length, and the ratios $\left(\sigma / \rho R_{0}\right)^{1 / 2}$ and $\sigma / R_{0}$ as velocity and pressure units. Restricting to axisymmetric configurations, continuity and inviscid momentum equations in the radial and axial directions are

$$
\begin{aligned}
& U_{r}+U / r+W_{z}=0, \\
& U_{t}+W\left(U_{z}-W_{r}\right)=-Q_{r}, \\
& W_{t}-U\left(U_{z}-W_{r}\right)=-Q_{z}-B,
\end{aligned}
$$

where $r$ and $z$ are the radial and axial coordinates, $U$ and $W$ are the radial and axial velocity components, and

$$
Q=P+\frac{1}{2}\left(U^{2}+W^{2}\right)
$$

is the stagnation pressure. Subscripts indicate hereafter derivation with respect to the independent variables. The boundary conditions [disks impenetrability, free surface anchoring to the sharp disks edges, and kinematic compatibility and equilibrium of normal stress at the free surface, defined as $r=F(z)]$ are

$$
\begin{aligned}
& W(r, \pm \Lambda, t)=0, \\
& F( \pm \Lambda, t)=1 \pm H, \\
& F_{t}(z, t)=U(F(z, t), z, t)-F_{z}(z, t) W(F(z, t), z, t),
\end{aligned}
$$

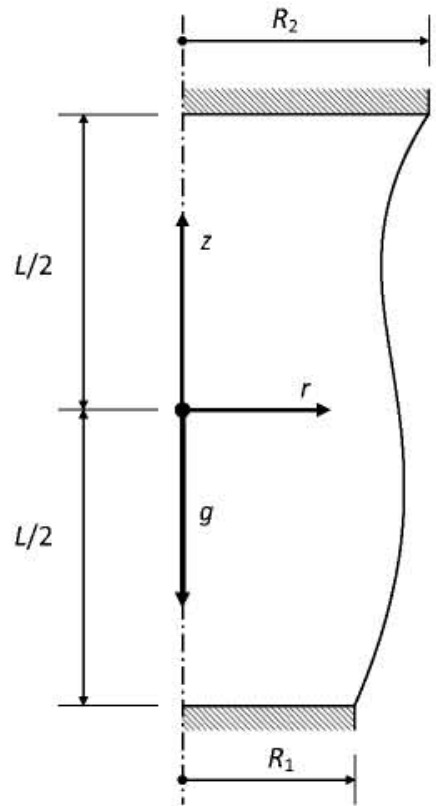

FIG. 2. A meridian section of the liquid bridge configuration.

$$
\begin{aligned}
& Q(F(z, t), z, t)-\frac{1}{2}\left[U^{2}(F(z, t), z, t)+W^{2}(F(z, t), z, t)\right] \\
& \quad+\mathrm{M}[F(z, t)]=0,
\end{aligned}
$$

where

$$
\mathrm{M}[F]=\frac{F F_{z z}-1-F_{z}^{2}}{F\left(1+F_{z}^{2}\right)^{3 / 2}},
$$

is the mean curvature of the free surface. For convenience, we also state volume preservation,

$$
\int_{-\Lambda}^{\Lambda} F^{2}(z, t) \mathrm{d} z=2 \Lambda\left(V_{0}+V\right),
$$

and the smoothness of the (axisymmetric) flow field at the axis,

$$
U(0, z, t)=W_{r}(0, z, t)=0 .
$$

Finally, suitable initial conditions for the velocity and the interface shape, satisfying Eqs. (2)-(12), should be imposed that are assumed to be compatible with the perturbation scheme below.

Quiescent steady states are such that $U_{0}=0,, W_{0}=0$, $Q_{0}=$ constant. Thus, invoking Eqs. (6)-(9), the steady free surface deformation $F_{0}$ is given by the following two point boundary value problem:

$$
\begin{aligned}
& \mathrm{M}\left[F_{0}(z)\right]+Q_{0}=0, \\
& F_{0}( \pm \Lambda)=1, \\
& \int_{-\Lambda}^{\Lambda} F^{2}(z, t) \mathrm{d} z=2 \Lambda V_{0} .
\end{aligned}
$$

The solution to this problem is well known and can be either written in terms of elliptic integrals ${ }^{11}$ or solved numerically. The instability limit of these is readily calculated imposing that the linearized problem around the steady states exhibits 


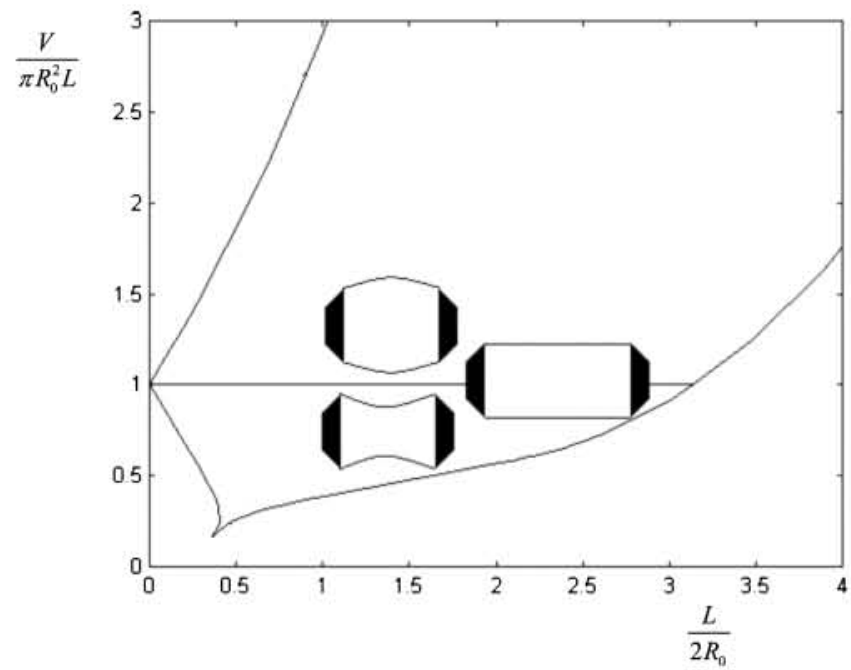

FIG. 3. Stability limits of liquid bridges between equal disks for zero Bond number. Sketches show stable liquid bridges with volumes larger, equal, and smaller than the corresponding to a cylinder.

a nontrivial solution (see, e.g., Martinez and Perales). ${ }^{39}$ It follows that the perturbed velocity components identically vanish, the perturbed pressure $q=Q-Q_{0}$ is spatially constant, and the perturbed free surface deformation, $f=F-F_{0}$, is given by the following linearized, second order, two point boundary value problem:

$$
\begin{aligned}
& \mathrm{M}^{\prime}\left[F_{0}(z)\right](f)+q=0, \\
& f( \pm \Lambda)=0, \\
& \int_{-\Lambda}^{\Lambda} F_{0}(z) f(z) \mathrm{d} z=0,
\end{aligned}
$$

where the linear operator $\mathrm{M}^{\prime}\left[F_{0}\right]$ is the Frechet derivative of the mean curvature operator (10), namely,

$$
\begin{aligned}
\mathrm{M}^{\prime}\left[F_{0}\right](f)= & \frac{1}{\left(1+F_{0 z}{ }^{2}\right)^{3 / 2}} f_{z z}+\left[\frac{F_{0 z}}{F_{0}\left(1+F_{0 z}{ }^{2}\right)^{3 / 2}}\right. \\
& \left.-\frac{3 F_{0 z} F_{0 z z}}{\left(1+F_{0 z}{ }^{2}\right)^{5 / 2}}\right] f_{z}+\frac{1}{F_{0}^{2}\left(1+F_{0 z}{ }^{2}\right)^{1 / 2}} f \\
= & \frac{1}{F_{0}} \frac{d}{d z}\left[\frac{F_{0} f_{z}}{\left(1+F_{0 z}{ }^{2}\right)^{3 / 2}}\right]+\frac{f}{F_{0}^{2}\left(1+F_{0 z}{ }^{2}\right)^{1 / 2}} .
\end{aligned}
$$

For each value of the slenderness this condition (and its counterpart for nonaxisymmetric perturbations) provides two threshold values of the volume, one lower and one upper, which will be called the instability limits below. These are plotted in Fig. 3, where the stable configurations are those in between of the instability limits. The upper instability limit is associated with nonaxisymmetric perturbations. The lower instability limit is associated with axisymmetric perturbations, which are either reflection symmetric on the plane $z=0$ if $\Lambda_{A} \cong 2.127>\Lambda>\Lambda_{C} \cong 0.361$, or antisymmetric in $z$ if $\Lambda>\Lambda_{A} \cong 2.127$. Thus, in the former case the breaking process is essentially symmetric and gives one smaller central drop and two larger lateral drops, while in the latter case, the liquid bridge breaks into two clearly different drops (with a third smaller one in between). If $\Lambda<\Lambda_{C}$, the unstable perturbations are again nonaxisymmetric, which are outside the scope of this paper. Now, at $\Lambda=\Lambda_{A}$ a mode interaction occurs between the symmetric and antisymmetric instabilities that requires considering symmetric and antisymmetric perturbations simultaneously; this mode interaction process is associated with a codimension two bifurcation, whose analysis is outside of the scope of this paper. Note that the symmetric and antisymmetric instability limits are tangent to each other at $\Lambda=\Lambda_{A}$.

Against this background, the main object of this paper is to describe both oscillations and breaking near the instability limits in a weakly nonlinear setting, namely, assuming that perturbations of the free surface deflection are small. For the sake of clarity, we anticipate here the main results:

(A) In the nonsymmetric case (near the lower instability limit in Fig. 3, as $\Lambda>\Lambda_{A}$ ), perturbations of the free surface are given by

$F(z, t)=F_{0}(z)+A(t) f(z)+\cdots$,

where $f(z) \sim 1$ is a solution of the linearized problems (16)-(18) and the mode amplitude $A(t)$ is small, namely,

$|A(t)| \ll 1$,

and given by the following forced Duffing equation:

$\mu_{1} \ddot{A}(t)+\widetilde{g}_{12} V A(t)+\widetilde{g}_{111} A^{3}(t)=\widetilde{g}_{3} H+\widetilde{g}_{4} B$,

which generally gives perturbations of one mode oscillations in conservative systems in the presence of reflection symmetry, and has been widely studied; see Nayfeh and Mook, ${ }^{40}$ Guckenheimer and Holmes, ${ }^{41}$ Moon, ${ }^{42}$ and Thompson and Stewart ${ }^{43}$ for comprehensive reviews of the associated literature. Here, dependence of the various coefficients on the small parameters $V, H$, and $B$, and the fact that nonlinearity is cubic, are anticipated from symmetry considerations, noting that the equation must be invariant under the symmetry group

$A \rightarrow-A, \quad H \rightarrow-H, \quad B \rightarrow-B$.

Assumptions (1) and (21) imply that the amplitude $A(t)$ evolves in a large timescale (compared with the capillary timescale used above for nondimensionalization). Equation (22) allows for a complete understanding of the dynamics near the instability limit.

(B) In the symmetric case (near the lower instability limit in Fig. 3 as $\Lambda_{A}>\Lambda>\Lambda_{C}$ ), perturbations of the free surface are given by the following quadratic Duffinglike equation:

$\mu_{2} \ddot{C}(t)+\widetilde{g}_{11} C^{2}(t)+\widetilde{g}_{2} V=\widetilde{g}_{33} H^{2}+\widetilde{g}_{34} B H+\widetilde{g}_{44} B^{2}$,

which also allows understanding the dynamics in this case. Again, dependence of the coefficients results from symmetry considerations, noting that Eq. (24) must be invariant under the symmetry group 
$H \rightarrow-H, \quad B \rightarrow-B$.

The remaining of the paper is organized as follows. The various coefficients appearing in Eqs. (22) and (24) will be calculated in Secs. III and IV, respectively, where two different scalings will be considered. Equations (22) and (24) will be used in Sec. V to elucidate the weakly nonlinear dynamics of the system near the instability limits, which in turn will allow us to explain some anomalous experimental behaviors and to make some predictions. These will be checked comparing with available experiments in literature in Sec. V. The paper ends with some concluding remarks, in Sec. VI.

\section{CASE $\Lambda>\Lambda_{A}$}

Two different scalings must be considered depending on the value of $\Lambda$. If $\Lambda>\Lambda_{\mathrm{A}}$ (with $\Lambda_{\mathrm{A}}=2.13 \cdots$, see Martínez and Perales) ${ }^{39}$ an order of magnitude analysis yields the appropriate scaling for both the parameters,

$$
V=\varepsilon^{2} v, \quad B=\varepsilon^{3} b, \quad H=\varepsilon^{3} h, \quad \tau=\varepsilon t,
$$

and the state variables. The latter are seen to be such that $U \sim W \sim\left(Q-Q_{0}\right) \sim\left(F-F_{0}\right) \sim \varepsilon$, where $Q_{0}$ and $F_{0}$ are given by Eqs. (13)-(15). Thus, perturbations of this steady state are expanded in powers of $\varepsilon$ as

$$
\begin{aligned}
& f=F_{0}(r, z)+\varepsilon f_{1}(z, t)+\varepsilon^{2} f_{2}(z, \tau)+\varepsilon^{3} f_{3}(z, \tau)+\cdots, \\
& q(r, z, \tau)=Q_{0}(r, z)+\varepsilon q_{1}(r, z, \tau)+\varepsilon^{2} q_{2}(r, z, \tau) \\
& +\varepsilon^{3} q_{3}(r, z, \tau)+\cdots, \\
& u(r, z, \tau)=\varepsilon u_{2}(r, z, \tau)+\varepsilon^{2} u_{3}(r, z, \tau)+\cdots, \\
& w(r, z, \tau)=\varepsilon w_{2}(r, z, \tau)+\varepsilon^{2} w_{3}(r, z, \tau)+\cdots .
\end{aligned}
$$

Substituting these into the governing equations and boundary conditions and setting to zero the coefficients of the various powers of $\varepsilon$ leads to the linear problems that are considered below. It must be noted that the continuity equation at order $\varepsilon^{k}$ couples with momentum equations at order $\varepsilon^{k+1}$.

\section{A. Order $\varepsilon$}

At this order, $q_{1}$ is readily seen to satisfy $q_{1 r}=q_{1 z}=0$, which means that $q_{1}$ is spatially constant (but may depend on time), and $f_{1}$ satisfies the linearized problems (16)-(18). Since this latter problem does not exhibit temporal derivatives, $f_{1}$ and $q_{1}$ can be written as

$$
f_{1}(z, \tau)=a(\tau) f_{a 1}(z), \quad q_{1}(r, z, \tau)=a(\tau) q_{a 1},
$$

where $f_{a 1}(z)$ and $q_{a 1}$ (which is constant) are still given by Eqs. (16)-(18). Note that this latter problem exhibits nontrivial solutions precisely at the instability limit in Fig. 3. In the present case $\left(\Lambda>\Lambda_{\mathrm{A}}\right)$ the nontrivial solutions are antisymmetric in $z$. It follows that

$$
f_{a 1}(z)=F_{0 z}(z), \quad q_{a 1}=0,
$$

up to a constant factor.

\section{B. Terms of order $\varepsilon^{2}$}

$$
\begin{aligned}
& q_{2 r}=0, \\
& q_{2 z}=0, \\
& \begin{aligned}
& \mathrm{M}^{\prime} {\left[F_{0}(z)\right]\left(f_{2}(z, \tau)\right)+q_{2}\left(F_{0}(z), z, \tau\right) } \\
& \quad=-\frac{1}{2} a^{2}(\tau) \mathrm{M}^{\prime \prime}\left[F_{0}(z)\right]\left(f_{a 1}(z), f_{a 1}(z)\right), \\
& f_{2}( \pm \Lambda, \tau)=0, \\
& \int_{-\Lambda}^{\Lambda} F_{0}(z) f_{2}(z, \tau) \mathrm{d} z=-\frac{a^{2}(\tau)}{2} \int_{-\Lambda}^{\Lambda} f_{a 1}^{2}(z) \mathrm{d} z+\Lambda v .
\end{aligned}
\end{aligned}
$$

As above, Eqs. (32) and (33) imply that $q_{2}$ depends only on $\tau$. And a look at the right hand side of Eqs. (34) and (36) suggests to split $f_{2}$ and $q_{2}$ as

$$
\begin{aligned}
& f_{2}(z, \tau)=a^{2}(\tau) \tilde{f}_{11}(z)+v \widetilde{f}_{2}(z), \\
& q_{2}(\tau)=a^{2}(\tau) \widetilde{q}_{11}+v \widetilde{q}_{2} .
\end{aligned}
$$

Substitution of these into Eqs. (34)-(36) leads to the following problems to calculate $\left(\tilde{f}_{11}, \widetilde{q}_{11}\right)$ and $\left(\tilde{f}_{2}, \widetilde{q}_{2}\right)$ :

$$
\begin{aligned}
& \mathbf{M}^{\prime}\left[F_{0}(z)\right]\left(\tilde{f}_{11}(z)\right)+\widetilde{q}_{11}=-\frac{1}{2} \mathbf{M}^{\prime \prime}\left[F_{0}(z)\right]\left(f_{a 1}(z), f_{a 1}(z)\right) \\
& \tilde{f}_{11}( \pm \Lambda)=0 \\
& \int_{-\Lambda}^{\Lambda} F_{0}(z) \tilde{f}_{11}(z) \mathrm{d} z=-\frac{1}{2} \int_{-\Lambda}^{\Lambda} f_{a 1}^{2}(z) \mathrm{d} z
\end{aligned}
$$

and

$$
\begin{aligned}
& \mathbf{M}^{\prime}\left[F_{0}(z)\right]\left(\tilde{f}_{2}(z)\right)+\tilde{q}_{2}=0, \\
& \tilde{f}_{2}( \pm \Lambda)=0,
\end{aligned}
$$

$$
\int_{-\Lambda}^{\Lambda} F_{0}(z) \tilde{f}_{2}(z) \mathrm{d} z=\Lambda
$$

Note that these two problems are singular, since their left hand sides coincide with the homogenous linear problems (16)-(18), which exhibits nontrivial solutions. But the forcing terms are reflection symmetric in $z$, while nontrivial solutions of the homogenous part are antisymmetric, which means that the problems above are solvable. The resulting solutions are of course nonunique since any solution of the homogenous problem can be added to a given solution. For convenience, we set

$$
\int_{-\Lambda}^{\Lambda} F_{0}(z) f_{a 1}(z) \tilde{f}_{11}(z) \mathrm{d} z=0,
$$




$$
\int_{-\Lambda}^{\Lambda} F_{0}(z) f_{a 1}(z) \tilde{f}_{2}(z) \mathrm{d} z=0 .
$$

Note that in the two above problems $f_{a 1}(z)$ is a known function [in fact, it is equal to $F_{0 z}(z)$ ]. These problems have been already solved analytically (in terms of elliptic integrals) by Gómez, Parra, and Perales. ${ }^{11}$ It should be noted that neither $\widetilde{q}_{11}$ nor $\widetilde{q}_{2}$ are needed to determine the dynamical behavior; only $\tilde{f}_{11}(z)$ and $\tilde{f}_{2}(z)$ need to be computed.

\section{Terms of order $\varepsilon^{3}$}

$$
\begin{aligned}
& u_{2 r}+u_{2} / r+w_{2 z}=0, \\
& q_{3 r}=-u_{2 \tau}, \\
& q_{3 z}=-w_{2 \tau}-b, \\
& w_{2}(r, \pm \Lambda, \tau)=0, \\
& u_{2}(0, z, \tau)=w_{2 r}(0, z, \tau)=0, \\
& u_{2}\left(F_{0}(z), z, \tau\right)-F_{0 z}(z) w_{2}\left(F_{0}(z), z, \tau\right)=\dot{a}(\tau) f_{a 1}(z), \\
& \mathbf{M}^{\prime}\left[F_{0}(z)\right]\left(f_{3}(z, \tau)\right)+q_{3}\left(F_{0}(z), z, \tau\right) \\
& =-a(\tau) \mathrm{M}^{\prime \prime}\left[F_{0}(z)\right]\left(f_{a 1}(z), f_{2}(z, \tau)\right) \\
& -a(\tau) f_{a 1}(z) q_{2 r}\left(F_{0}(z), z, \tau\right) \\
& -\frac{1}{6} a^{3}(\tau) \mathbf{M}^{\prime \prime \prime}\left[F_{0}(z)\right]\left(f_{a 1}(z), f_{a 1}(z), f_{a 1}(z)\right), \\
& f_{3}( \pm \Lambda, \tau)= \pm h, \\
& \int_{-\Lambda}^{\Lambda} F_{0}(z) f_{3}(z, \tau) \mathrm{d} z=-a(\tau) \int_{-\Lambda}^{\Lambda} f_{a 1}(z) f_{2}(z, \tau) \mathrm{d} z .
\end{aligned}
$$

Equations (46) and (47) allow for eliminating $u_{2}$ and $w_{2}$ in Eqs. (45)-(53). A further look at the boundary condition (50) suggests rewriting $q_{3}$ as

$$
q_{3}(r, z, \tau)=\ddot{a}(\tau) \Phi(r, z)-b z,
$$

which substituted into Eqs. (45)-(53) yields

$$
\begin{aligned}
& \Phi_{r r}+\Phi_{r} / r+\Phi_{z z}=0, \\
& \Phi_{z}(r, \pm \Lambda)=0, \\
& \Phi_{r}(0, z)=\Phi_{r z}(0, z)=0, \\
& \Phi_{r}\left(F_{0}(z), z\right)-F_{0 z}(z) \Phi_{z}\left(F_{0}(z), z\right)=f_{a 1}(z) . \\
& \mathrm{M}^{\prime}\left[F_{0}(z)\right]\left(f_{3}(z, \tau)\right)+\ddot{a}(\tau) \Phi\left(F_{0}(z), z\right)-b z \\
& \quad=-a(\tau) v \mathrm{M}^{\prime \prime}\left[F_{0}(z)\right]\left(f_{a 1}(z), \tilde{f}_{2}(z)\right) \\
& \quad-a^{3}(\tau) \mathrm{M}^{\prime \prime}\left[F_{0}(z)\right]\left(f_{a 1}(z), \tilde{f}_{11}(z)\right) \\
& \quad-\frac{1}{6} a^{3}(\tau) \mathrm{M}^{\prime \prime \prime}\left[F_{0}(z)\right]\left(f_{a 1}(z), f_{a 1}(z), f_{a 1}(z)\right), \\
& f_{3}( \pm \Lambda, \tau)= \pm h, \\
& \int_{-\Lambda}^{\Lambda} F_{0}(z) f_{3}(z, \tau) \mathrm{d} z \\
& \quad=-a(\tau) v \int_{-\Lambda}^{\Lambda} f_{a 1}(z) \tilde{f}_{2}(z) \mathrm{d} z \\
& \quad-a^{3}(\tau) \int_{-\Lambda}^{\Lambda} f_{a 1}(z) \tilde{f}_{11}(z) \mathrm{d} z .
\end{aligned}
$$

Solving Eqs. (54)-(57) for $\Phi$ and substituting such solution into Eqs. (58)-(60) yields a two point boundary value problem to determine $f_{3}$. Such problem needs not be solved. Instead we only apply a solvability condition, noting that its homogenous part exhibits nontrivial solutions [see Eqs. (16)-(18) above]. Such solvability condition is readily obtained multiplying Eq. (58) by $F_{0}(z) f_{a 1}(z)$ and integrating the resulting equation in $-\Lambda \leq z \leq \Lambda$ to obtain

$$
\begin{aligned}
\int_{-\Lambda}^{\Lambda} \mathrm{M}^{\prime}\left[F_{0}(z)\right]\left(f_{3}(z, \tau)\right) F_{0}(z) f_{a 1}(z) \mathrm{d} z+\ddot{a}(\tau) \int_{-\Lambda}^{\Lambda} \Phi\left(F_{0}(z), z\right) F_{0}(z) f_{a 1}(z) \mathrm{d} z-b \int_{-\Lambda}^{\Lambda} z F_{0}(z) f_{a 1}(z) \mathrm{d} z \\
=-a(\tau) v \int_{-\Lambda}^{\Lambda} \mathrm{M}^{\prime \prime}\left[F_{0}(z)\right]\left(f_{a 1}(z), \tilde{f}_{2}(z)\right) F_{0}(z) f_{a 1}(z) \mathrm{d} z \\
\quad-a^{3}(\tau) \int_{-\Lambda}^{\Lambda}\left\{\mathrm{M}^{\prime \prime}\left[F_{0}(z)\right]\left(f_{a 1}(z), \tilde{f}_{11}(z)\right)+\frac{1}{6} \mathrm{M}^{\prime \prime \prime}\left[F_{0}(z)\right]\left(f_{a 1}(z), f_{a 1}(z), f_{a 1}(z)\right)\right\} F_{0}(z) f_{a 1}(z) \mathrm{d} z,
\end{aligned}
$$




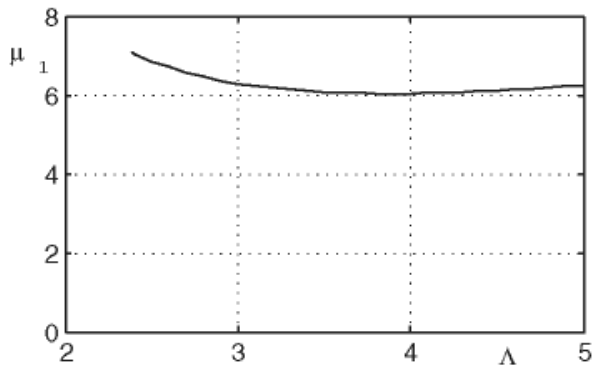

FIG. 4. Mass coefficient for the antisymmetric mode, $\mu_{1}$.

where the unknown $f_{3}$ is still present in the first integral. But this can be eliminated using the following expression, which is readily obtained integrating by parts,

$$
\begin{aligned}
& \int_{-\Lambda}^{\Lambda} \mathrm{M}^{\prime}\left[F_{0}(z)\right](g(z)) F_{0}(z) f_{a 1}(z) \mathrm{d} z \\
& \quad=-\left.\frac{F_{0}(z) f_{a 1 z}(z) g(z)}{F_{0}(z)\left(1+F_{0 z}^{2}(z)\right)^{3 / 2}}\right|_{-\Lambda} ^{\Lambda}-q_{1} \int_{-\Lambda}^{\Lambda} F_{0}(z) g(z) \mathrm{d} z
\end{aligned}
$$

Here, $g$ is any smooth enough function. Setting $g=f_{3}$ yields

$$
\begin{gathered}
\int_{-\Lambda}^{\Lambda} \mathbf{M}^{\prime}\left[F_{0}(z)\right]\left(f_{3}(z, \tau)\right) F_{0}(z) f_{a 1}(z) \mathrm{d} z \\
\quad=-2 h \frac{F_{0}(\Lambda) f_{a 1 z}(\Lambda)}{F_{0}(\Lambda)\left(1+F_{0 z}^{2}(\Lambda)\right)^{3 / 2}} .
\end{gathered}
$$

A further substitution into Eq. (61) provides the following Duffing equation:

$$
\mu_{1} \ddot{a}(\tau)+\widetilde{g}_{12} v a(\tau)+\widetilde{g}_{111} a^{3}(\tau)=\widetilde{g}_{3} h+\widetilde{g}_{4} b,
$$

with

$$
\begin{aligned}
& \mu_{1}=\int_{-\Lambda}^{\Lambda} \Phi\left(F_{0}(z), z\right) F_{0}(z) f_{a 1}(z) \mathrm{d} z, \\
& \tilde{g}_{12}=\int_{-\Lambda}^{\Lambda} \mathbf{M}^{\prime \prime}\left[F_{0}(z)\right]\left(f_{a 1}, \tilde{f}_{2}\right) F_{0}(z) f_{a 1}(z) \mathrm{d} z, \\
& \tilde{g}_{111}=\int_{-\Lambda}^{\Lambda}\left\{\mathbf{M}^{\prime \prime}\left[F_{0}(z)\right]\left(f_{a 1}, \tilde{f}_{11}\right)\right. \\
& \left.+\frac{1}{6} \mathbf{M}^{\prime \prime \prime}\left[F_{0}(z)\right]\left(f_{a 1}, f_{a 1}, f_{a 1}\right)\right\} F_{0}(z) f_{a 1}(z) \mathrm{d} z, \\
& \widetilde{g}_{3}=2 \frac{F_{0}(\Lambda) f_{a 1 z}(\Lambda)}{F_{0}(\Lambda)\left[1+F_{0 z}^{2}(\Lambda)\right]^{3 / 2}}, \\
& \widetilde{g}_{4}=\int_{-\Lambda}^{\Lambda} z F_{0}(z) f_{a 1}(z) \mathrm{d} z .
\end{aligned}
$$

The mass coefficient, $\mu_{1}$, is plotted versus slenderness in Fig. 4 and the remaining (stability) coefficients values are considered in Fig. 5.

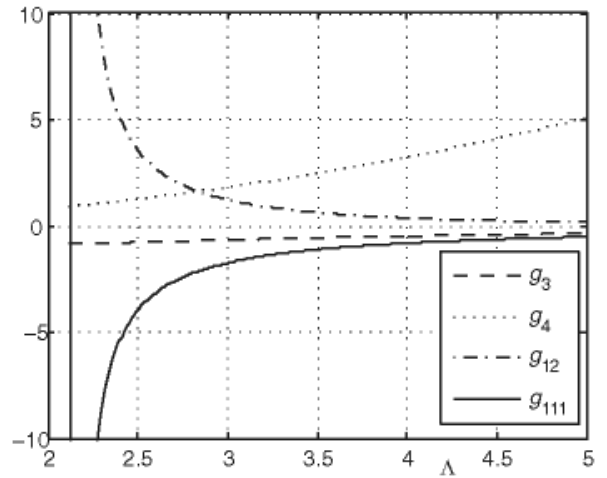

FIG. 5. Stability coefficients. The different coefficients have been calculated for $\Lambda>\Lambda_{\mathrm{A}}$, shown by a vertical line in the figure.

In the original dimensionless variables, ignoring the rescaling made above and defining $A(t)=\varepsilon a(\tau)$, namely, setting

$$
F(z, t)=F_{0}(z)+A(t) f_{a 1}(z),
$$

with $F_{0}(z)$ and $f_{\mathrm{al}}(z)$ as given by the solutions of the problem above, the time evolution of $A(t)$ is governed by

$$
\mu_{1} \frac{\mathrm{d}^{2} A(t)}{\mathrm{d} t^{2}}+\widetilde{g}_{12} V A(t)+\widetilde{g}_{111} A^{3}(t)=\widetilde{g}_{3} H+\widetilde{g}_{4} B,
$$

which is a standard Duffing equation. Here $V, H$, and/or $B$ may be slowly varying with time (although, for most applications only $B$ will be considered as variable). Note that the different mass and stability coefficients depend only functions on $\Lambda$.

\section{CASE $\Lambda<\Lambda_{\text {A }}$}

The case $\Lambda_{\mathrm{C}}<\Lambda<\Lambda_{\mathrm{A}}$ is now analyzed by following a similar procedure used for the case $\Lambda>\Lambda_{\mathrm{A}}$, thus only the differences with the previous case will be highlighted. The appropriate scaling on the parameters and the time variable is now

$$
V=\delta^{2} v, \quad B=\delta b, \quad H=\delta h, \quad \tau=\delta^{1 / 2} t .
$$

Inspection on the governing equations and boundary conditions (2)-(9) shows that $U \sim W \sim \delta^{3 / 2}$ and $Q-Q_{0} \sim F-F_{0}$ $\sim \delta$, which means that the dependent variables must be expanded as

$$
\begin{aligned}
& U(r, z, t)=\delta^{3 / 2} u_{3 / 2}(r, z, \tau)+\cdots, \\
& W(r, z, t)=\delta^{3 / 2} w_{3 / 2}(r, z, \tau)+\cdots, \\
& Q(r, z, t)=Q_{0}+\delta q_{1}(r, z, \tau)+\delta^{2} q_{2}(r, z, \tau)+\cdots, \\
& F(z, t)=F_{0}(z)+\delta f_{1}(z, \tau)+\delta^{2} f_{2}(z, \tau)+\cdots,
\end{aligned}
$$

where the steady state solution $\left(Q_{0}, F_{0}\right)$ is again given by Eqs. (13)-(15). Substituting these expansions into Eqs. (2)-(9) and setting to zero the coefficients of $\delta, \delta^{3 / 2}$, and $\delta^{2}$ leads to the three problems that are considered below. 


\section{A. Order $\delta$}

$q_{1 r}=0$,

$q_{1 z}=-b$,

$\mathrm{M}^{\prime}\left[F_{0}(z)\right]\left(f_{1}(z, \tau)\right)+q\left(F_{0}(z), z, \tau\right)=0$,

$f_{1}( \pm \Lambda, \tau)= \pm h$

$$
\int_{-\Lambda}^{\Lambda} F_{0}(z) f_{1}(z, \tau) \mathrm{d} z=0
$$

The most general solution to this linear problem can be written as

$$
\begin{aligned}
& f_{1}(z, \tau)=c(\tau) f_{s 1}(z)+h \tilde{f}_{3}(z)+b \tilde{f}_{4}(z), \\
& q_{1}(r, z, \tau)=c(\tau) q_{s 1}-b z+h \widetilde{q}_{3}+b \widetilde{q}_{4},
\end{aligned}
$$

where $f_{s 1}$ and $q_{s 1}$ (which is constant) are given by the linearized problems (16)-(18). The remaining terms, $\tilde{f}_{3}, \tilde{q}_{3}, \tilde{f}_{4}$, and $\widetilde{q}_{4}$, are calculated from the following problems (already solved by Gómez, Parra, and Perales): ${ }^{11}$

$$
\begin{aligned}
& \mathrm{M}^{\prime}\left[F_{0}(z)\right]\left(\tilde{f}_{3}(z)\right)+\tilde{q}_{3}=0, \\
& \tilde{f}_{3}( \pm \Lambda)= \pm 1 \\
& \int_{-\Lambda}^{\Lambda} F_{0}(z) \tilde{f}_{3}(z) \mathrm{d} z=0, \\
& \mathrm{M}^{\prime}\left[F_{0}(z)\right]\left(\tilde{f}_{4}(z)\right)+\widetilde{q}_{4}=z, \\
& \tilde{f}_{4}( \pm \Lambda)=0 \\
& \int_{-\Lambda}^{\Lambda} F_{0}(z) \tilde{f}_{4}(z) \mathrm{d} z=0,
\end{aligned}
$$

As in the antisymmetric case, these two latter problems are singular, since their left hand sides coincide with the homogenous linear problems (16)-(18), which exhibits nontrivial solutions. But the forcing terms are reflection antisymmetric in $z$, while nontrivial solutions of homogenous part are symmetric, meaning that the problems above are solvable. The resulting solutions are of course nonunique since any solution of the homogenous problem can be added to a given solution. For convenience, a unique solution is selected setting

$$
\begin{aligned}
& \int_{-\Lambda}^{\Lambda} F_{0}(z) f_{s 1}(z) \tilde{f}_{3}(z) \mathrm{d} z=0, \\
& \int_{-\Lambda}^{\Lambda} F_{0}(z) f_{s 1}(z) \tilde{f}_{4}(z) \mathrm{d} z=0 .
\end{aligned}
$$

\section{B. Order $\delta^{3 / 2}$}

$$
\begin{aligned}
& u_{3 / 2 r}+u_{3 / 2} / r+w_{3 / 2 z}=0 \\
& u_{3 / 2 \tau}=-q_{2 r} \\
& w_{3 / 2 \tau}=-q_{2 z} \\
& w_{3 / 2}(r, \pm \Lambda, \tau)=0 \\
& u_{3 / 2}(0, z, \tau)=w_{3 / 2 r}(0, z, \tau)=0 \\
& u_{3 / 2}\left(F_{0}(z), z, \tau\right)-F_{0 z}(z) w_{3 / 2}\left(F_{0}(z), z, \tau\right)=\dot{c}(\tau) f_{s 1}(z) .
\end{aligned}
$$

\section{Order $\delta^{2}$}

$$
\begin{aligned}
& \mathrm{M}^{\prime}\left[F_{0}(z)\right]\left(f_{2}(z, \tau)\right)+q_{2}\left(F_{0}(z), z, \tau\right) \\
& =-\frac{1}{2} \mathrm{M}^{\prime \prime}\left[F_{0}(z)\right]\left(c(\tau) f_{s 1}(z)+h \tilde{f}_{3}(z)\right. \\
& \left.\quad+b \tilde{f}_{4}(z), c(\tau) f_{s 1}(z)+h \tilde{f}_{3}(z)+b \tilde{f}_{4}(z)\right), \\
& f_{2}( \pm \Lambda, \tau)=0, \\
& \int_{-\Lambda}^{\Lambda} F_{0}(z) f_{2}(z, \tau) \mathrm{d} z \\
& \quad=-\frac{1}{2} \int_{-\Lambda}^{\Lambda}\left(c(\tau) f_{s 1}(z)+h \tilde{f}_{3}(z)+b \tilde{f}_{4}(z)\right)^{2} \mathrm{~d} z+\Lambda v .
\end{aligned}
$$

Now, $u_{3 / 2}$ and $w_{3 / 2}$ can be eliminated by differentiating with respect to $\tau$ in Eqs. (86) and (87), and substituting into Eqs. $(85)$ and (88)-(90), which yield

$$
\begin{aligned}
& \frac{\partial}{\partial \tau}\left(q_{2 r r}+q_{2 r} / r+q_{2 z z}\right)=0, \\
& q_{2 z}(r, \pm \Lambda, \tau)=0, \\
& q_{2 r}(0, z, \tau)=q_{2 z r}(0, z, \tau)=0, \\
& q_{2 r}\left(F_{0}(z), z, \tau\right)-F_{0 z}(z) q_{2 z}\left(F_{0}(z), z, \tau\right)=\ddot{c}(\tau) f_{s 1}(z) .
\end{aligned}
$$

These equations suggest setting

$$
q_{2}(r, z, \tau)=\ddot{c}(\tau) \Phi(r, z),
$$

which substituted into Eqs. (94)-(97) provides the following problem for $\Phi$ [identical to that solved for $\Lambda>\Lambda_{\mathrm{A}}$ except for the new functions $F_{0}(z)$ and $\left.f_{s 1}(z)\right]$

$$
\begin{aligned}
& \Phi_{r r}+\Phi_{r} / r+\Phi_{z z}=0, \\
& \Phi_{z}(r, \pm \Lambda)=0, \\
& \Phi_{r}(0, z)=\Phi_{r z}(0, z)=0, \\
& \Phi_{r}\left(F_{0}(z), z\right)-F_{0 z}(z) \Phi_{z}\left(F_{0}(z), z\right)=f_{s 1}(z) .
\end{aligned}
$$

A further substitution into Eqs. (91)-(93) leads to 


$$
\begin{aligned}
& \mathrm{M}^{\prime}\left[F_{0}(z)\right]\left(f_{2}(z, \tau)\right)+\ddot{c}(\tau) \Phi\left(F_{0}(z), z\right)=-\frac{1}{2} c^{2}(\tau) \mathbf{M}^{\prime \prime}\left[F_{0}(z)\right]\left(f_{s 1}(z), f_{s 1}(z)\right)-\frac{1}{2} h^{2} \mathbf{M}^{\prime \prime}\left[F_{0}(z)\right]\left(\tilde{f}_{3}(z), \tilde{f}_{3}(z)\right) \\
& \quad-\frac{1}{2} b^{2} \mathbf{M}^{\prime \prime}\left[F_{0}(z)\right]\left(\tilde{f}_{4}(z), \tilde{f}_{4}(z)\right)-c(\tau) h \mathbf{M}^{\prime \prime}\left[F_{0}(z)\right]\left(f_{s 1}(z), \tilde{f}_{3}(z)\right) \\
& \quad-c(\tau) b \mathbf{M}^{\prime \prime}\left[F_{0}(z)\right]\left(f_{s 1}(z), \tilde{f}_{4}(z)\right)-h b \mathbf{M}^{\prime \prime}\left[F_{0}(z)\right]\left(\tilde{f}_{3}(z), \tilde{f}_{4}(z)\right) \\
& f_{2}( \pm \Lambda, \tau)=0 \\
& \int_{-\Lambda}^{\Lambda} F_{0}(z) f_{2}(z, \tau) \mathrm{d} z=-\frac{1}{2} \int_{-\Lambda}^{\Lambda}\left[c(\tau) f_{s 1}(z)+h \tilde{f}_{3}(z)+b \tilde{f}_{4}(z)\right]^{2} \mathrm{~d} z+\Lambda v \\
& \int_{-\Lambda}^{\Lambda} F_{0}(z) f_{s 1}(z) f_{2}(z, \tau) \mathrm{d} z=0 .
\end{aligned}
$$

As in last section, this problem needs not be solved. Instead, a solvability condition is applied that results from integrating the product of Eq. (102) times $F_{0}(z) f_{s 1}(z)$ between $-\Lambda$ and $\Lambda$, which yields

$$
\begin{aligned}
\int_{-\Lambda}^{\Lambda} \mathrm{M}^{\prime} & {\left[F_{0}(z)\right]\left(f_{2}(z, \tau)\right) F_{0}(z) f_{s 1}(z) \mathrm{d} z+\ddot{c}(\tau) \int_{-\Lambda}^{\Lambda} \Phi\left(F_{0}(z), z\right) F_{0}(z) f_{s 1}(z) \mathrm{d} z } \\
= & -\frac{1}{2} c^{2}(\tau) \int_{-\Lambda}^{\Lambda} \mathrm{M}^{\prime \prime}\left[F_{0}(z)\right]\left(f_{s 1}(z), f_{s 1}(z)\right) F_{0}(z) f_{s 1}(z) \mathrm{d} z-\frac{1}{2} h^{2} \int_{-\Lambda}^{\Lambda} \mathrm{M}^{\prime \prime}\left[F_{0}(z)\right]\left(\tilde{f}_{3}(z), \tilde{f}_{3}(z)\right) F_{0}(z) f_{s 1}(z) \mathrm{d} z \\
& -\frac{1}{2} b^{2} \int_{-\Lambda}^{\Lambda} \mathrm{M}^{\prime \prime}\left[F_{0}(z)\right]\left(\tilde{f}_{4}(z), \tilde{f}_{4}(z)\right) F_{0}(z) f_{s 1}(z) \mathrm{d} z-c(\tau) h \int_{-\Lambda}^{\Lambda} \mathrm{M}^{\prime \prime}\left[F_{0}(z)\right]\left(f_{s 1}(z), \tilde{f}_{3}(z)\right) F_{0}(z) f_{s 1}(z) \mathrm{d} z \\
& -c(\tau) b \int_{-\Lambda}^{\Lambda} \mathrm{M}^{\prime \prime}\left[F_{0}(z)\right]\left(f_{s 1}(z), \tilde{f}_{4}(z)\right) F_{0}(z) f_{s 1}(z) \mathrm{d} z-h b \int_{-\Lambda}^{\Lambda} \mathrm{M}^{\prime \prime}\left[F_{0}(z)\right]\left(\tilde{f}_{3}(z), \tilde{f}_{4}(z)\right) F_{0}(z) f_{s 1}(z) \mathrm{d} z
\end{aligned}
$$

Calculation of the integral in the left hand side apparently requires solving problems (102)-(105). Nevertheless, by using the above mentioned property (62) of the operator (repeated here)

$$
\begin{aligned}
& \int_{-\Lambda}^{\Lambda} \mathrm{M}^{\prime}\left[F_{0}(z)\right](g(z)) F_{0}(z) f_{s 1}(z) \mathrm{d} z \\
& \quad=-\left.\frac{F_{0}(z) f_{s 1 z}(z) g(z)}{F_{0}(z)\left[1+F_{0 z}^{2}(z)\right]^{3 / 2}}\right|_{-\Lambda} ^{\Lambda}-q_{1} \int_{-\Lambda}^{\Lambda} F_{0}(z) g(z) \mathrm{d} z
\end{aligned}
$$

and setting $g(z)=f_{2}(z)$, using the boundary condition (92) and the volume condition (93)

$$
\begin{aligned}
\int_{-\Lambda}^{\Lambda} \mathrm{M}^{\prime}\left[F_{0}(z)\right]\left(f_{2}(z, \tau)\right) F_{0}(z) f_{s 1}(z) \mathrm{d} z \\
=-q_{1} \int_{-\Lambda}^{\Lambda} F_{0}(z) f_{2}(z, \tau) \mathrm{d} z \\
=\frac{q_{1}}{2}\left[\int_{-\Lambda}^{\Lambda}\left[c(\tau) f_{s 1}(z)+h \tilde{f}_{3}(z)+b \tilde{f}_{4}(z)\right]^{2} \mathrm{~d} z-2 \Lambda v\right] \\
=\frac{q_{1}}{2}\left\{c^{2}(\tau) \int_{-\Lambda}^{\Lambda} f_{s 1}^{2}(z) \mathrm{d} z+h^{2} \int_{-\Lambda}^{\Lambda} \tilde{f}_{3}^{2}(z) \mathrm{d} z+b^{2} \int_{-\Lambda}^{\Lambda} \tilde{f}_{4}^{2}(z) \mathrm{d} z\right\} \\
\quad+q_{1}\left[h b \int_{-\Lambda}^{\Lambda} \tilde{f}_{3}(z) \tilde{f}_{4}(z) \mathrm{d} z+c(\tau) h \int_{-\Lambda}^{\Lambda} f_{s 1}(z) \tilde{f}_{3}(z) \mathrm{d} z+c(\tau) b \int_{-\Lambda}^{\Lambda} f_{s 1}(z) \tilde{f}_{4}(z) \mathrm{d} z\right]-q_{1} \Lambda v
\end{aligned}
$$




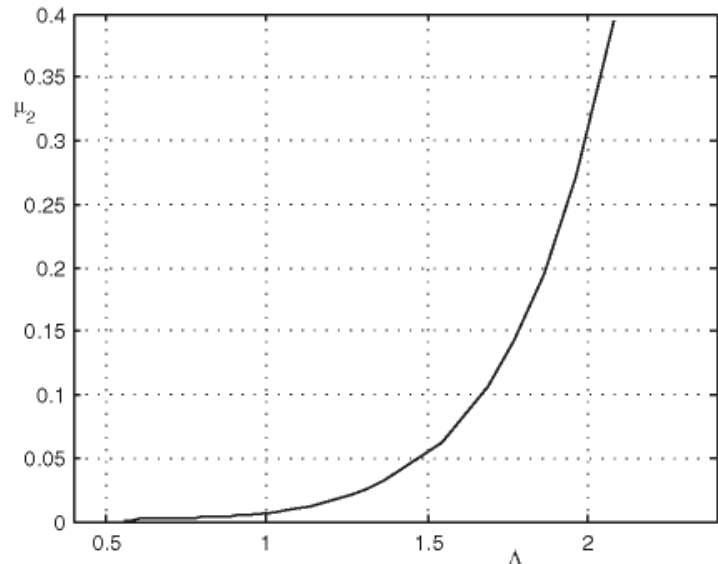

FIG. 6. Mass coefficient for the antisymmetric mode, $\mu_{2}$.

Equation (106) reduces to

$$
\begin{aligned}
& \mu_{2} \ddot{c}(\tau)+\widetilde{g}_{11} c^{2}(\tau)+\widetilde{g}_{2} v \\
& \quad=\widetilde{g}_{33} h^{2}+\widetilde{g}_{34} b h+\widetilde{g}_{44} b^{2}+\widetilde{g}_{13} c(\tau) h+\widetilde{g}_{14} c(\tau) b,
\end{aligned}
$$

with

$$
\begin{aligned}
\mu_{2}= & \int_{-\Lambda}^{\Lambda} \Phi\left(F_{0}(z), z\right) F_{0}(z) f_{s 1}(z) \mathrm{d} z, \\
\widetilde{g}_{11}= & \frac{1}{2} \int_{-\Lambda}^{\Lambda} \mathrm{M}^{\prime \prime}\left[F_{0}(z)\right]\left(f_{s 1}(z), f_{s 1}(z)\right) F_{0}(z) f_{s 1}(z) \mathrm{d} z \\
& +\frac{q_{1}}{2} \int_{-\Lambda}^{\Lambda} f_{s 1}^{2}(z) \mathrm{d} z,
\end{aligned}
$$$$
\widetilde{g}_{2}=-q_{1} \Lambda,
$$$$
\widetilde{g}_{33}=-\frac{1}{2} \int_{-\Lambda}^{\Lambda} \mathrm{M}^{\prime \prime}\left[F_{0}(z)\right]\left(\tilde{f}_{3}(z), \tilde{f}_{3}(z)\right) F_{0}(z) f_{s 1}(z) \mathrm{d} z
$$$$
-\frac{q_{1}}{2} \int_{-\Lambda}^{\Lambda} \tilde{f}_{3}^{2}(z) \mathrm{d} z
$$$$
\widetilde{g}_{34}=-\int_{-\Lambda}^{\Lambda} \mathbf{M}^{\prime \prime}\left[F_{0}(z)\right]\left(\tilde{f}_{3}(z), \tilde{f}_{4}(z)\right) F_{0}(z) f_{s 1}(z) \mathrm{d} z
$$$$
-q_{1} \int_{-\Lambda}^{\Lambda} \tilde{f}_{3}(z) \tilde{f}_{4}(z) \mathrm{d} z
$$$$
\tilde{g}_{44}=-\frac{1}{2} \int_{-\Lambda}^{\Lambda} \mathbf{M}^{\prime \prime}\left[F_{0}(z)\right]\left(\tilde{f}_{4}(z), \tilde{f}_{4}(z)\right) F_{0}(z) f_{s 1}(z) \mathrm{d} z
$$$$
-\frac{q_{1}}{2} \int_{-\Lambda}^{\Lambda} \widetilde{f}_{4}^{2}(z) \mathrm{d} z,
$$$$
\tilde{g}_{13}=-\int_{-\Lambda}^{\Lambda} \mathbf{M}^{\prime \prime}\left[F_{0}(z)\right]\left(f_{s 1}(z), \tilde{f}_{3}(z)\right) F_{0}(z) f_{s 1}(z) \mathrm{d} z
$$$$
-q_{1} \int_{-\Lambda}^{\Lambda} f_{s 1}(z) \tilde{f}_{3}(z) \mathrm{d} z=0,
$$

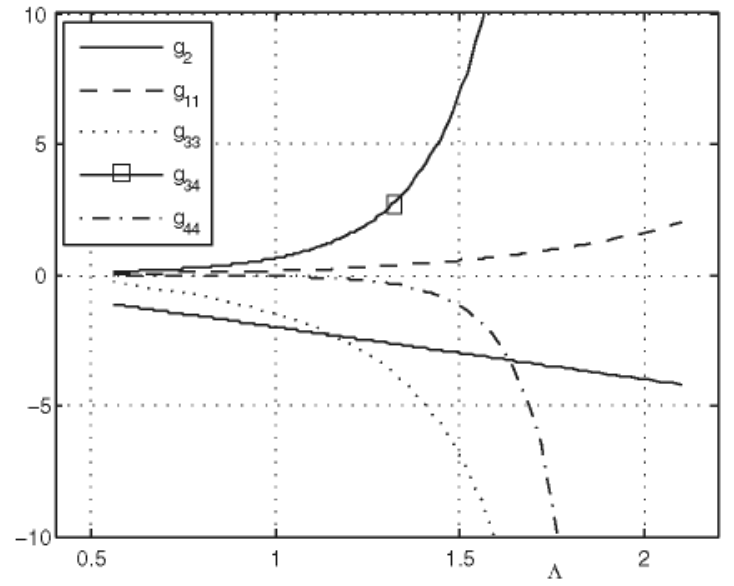

FIG. 7. Stability coefficients.

$$
\begin{aligned}
\tilde{g}_{14}= & -\int_{-\Lambda}^{\Lambda} \mathrm{M}^{\prime \prime}\left[F_{0}(z)\right]\left(f_{s 1}(z), \tilde{f}_{4}(z)\right) F_{0}(z) f_{s 1}(z) \mathrm{d} z \\
& -q_{1} \int_{-\Lambda}^{\Lambda} f_{s 1}(z) \tilde{f}_{4}(z) \mathrm{d} z=0,
\end{aligned}
$$

where the last two coefficients, $\widetilde{g}_{13}$ and $\widetilde{g}_{14}$, are zero due to the symmetries of the problem. The mass coefficient, $\mu_{2}$, is plotted in Fig. 6 and the different stability coefficients in Fig. 7.

Thus, the final equation governing the weakly non linear dynamics in this case is

$$
\mu_{2} \ddot{c}(\tau)+\widetilde{g}_{11} c^{2}(\tau)+\widetilde{g}_{2} v=\widetilde{g}_{33} h^{2}+\widetilde{g}_{34} b h+\widetilde{g}_{44} b^{2} .
$$

In the original dimensionless variables, defining $C(t)=\delta c(\tau)$ and using $F(z, t)=F_{0}(z)+C(t) f_{s 1}(z)$, with $F_{0}(z)$ and $f_{s 1}(z)$ given by the solutions of the problem above, the equation describing the evolution of the amplitude of the deformation is

$$
\mu_{2} \frac{\mathrm{d}^{2} C(t)}{\mathrm{d} t^{2}}+\widetilde{g}_{11} C^{2}(t)+\widetilde{g}_{2} V=\widetilde{g}_{33} H^{2}+\widetilde{g}_{34} B H+\widetilde{g}_{44} B^{2} .
$$

As in the last section, $V, H$, and/or $B$ may be allowed to be slowly varying functions of time and the different coefficients depend only on $\Lambda$.

\section{COMPARISON WITH EXPERIMENTS}

The model developed above applies to general (not necessarily cylindrical) configurations near to the stability limit. The Duffing or Duffing-like amplitude equations obtained above provides both the small nonlinear oscillations and the breaking process that occur under small excitation in the stable and unstable sides of the instability limit, respectively. The assumption that viscous dissipation is small is quite appropriate for experiments with ordinary liquids in microgravity conditions, but not to experiments in microzones, where viscous dissipation is significant. Microzones exhibit the ad- 


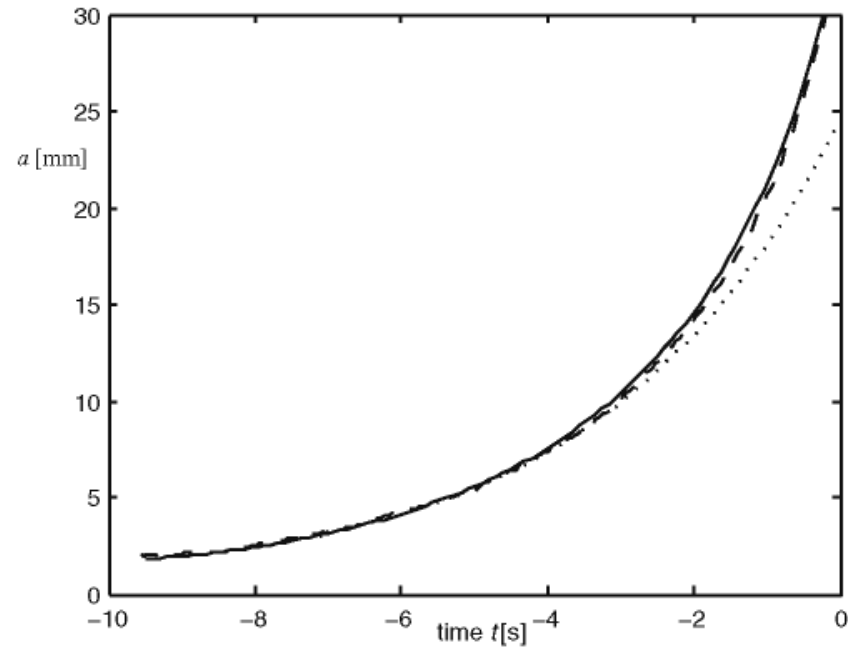

FIG. 8. Comparison of the diverging amplitude of the amphora-type deformation: experimental points (solid line), with the results of integrating Eq. (64) (dashed line). To better see the nonlinear terms effect, the integration has been repeated (dotted line) without including the term in $a^{3}$ (i.e., setting $\widetilde{g}_{111}=0$ instead of the actual value taken from Fig. 5 for that slenderness). The amplitude of the maximum deformation and the time are here plotted in physical units.

ditional difficulty that the experimental precision is limited, which makes it difficult to identify and measure small perturbations.

Only a few experimental results are available in actual microgravity conditions (to experimentally check the results), and none of them deal with noncylindrical configurations. Fortunately, some experimental results from past space experiments are available and can be used for validating results, at least in the limit of nearly cylindrical configurations. An unexpected breakage occurred during the second run of an experiment on the stability of liquid columns (STACO), performed in 1993, in the ESA Advanced Fluid Physics Module (located within the German WerkstoffLabor) ${ }^{25,44}$ on board Spacelab D-2. The experimental run imposed a slow disk separation, at constant volume, in a long liquid column (final slenderness was $\Lambda=3.13$ but with a volume corresponding to a cylinder at the starting slenderness). In order to check the ability of Eq. (64) to reproduce this experimental run, we integrate Eq. (64) from the end-of-stretching state (i.e., from $t_{0}=-9.5 \mathrm{~s}$ ) to $t=0 \mathrm{~s}$ (the breaking instant). At $t=t_{0}$, we impose the measured initial conditions, $a\left(t_{0}\right)$ $=0.026(0.4 \mathrm{~mm}, 1.8$ pixels in the image $)$ and $\dot{a}\left(t_{0}\right)=0.0016$ $(0.06 \mathrm{~mm} / \mathrm{s}, 0.3$ pixels $/ \mathrm{s})$. The result (plotted with dashed line) is compared in Fig. 8 with the actual experimental curve (solid line). Note that the experimental run is rather well approximated. In order to illustrate the role of the cubic nonlinear term in Eq. (64), the result of integrating Eq. (64) with the cubic term ignored is also plotted with dotted line.

For $\Lambda=\pi, V_{0}=1$ the present model coincides with its counterpart obtained from the well known Cosserat model $^{34,38}$ In particular for both models the values of the coefficients of $v, a$, and $a^{3}$ in Eq. (64) coincide but there is a small difference in the value of the coefficient in $\mathrm{d}^{2} a / \mathrm{d} t^{2}$ (a value of 6.25 for the Cosserat model and of 6.20 in the

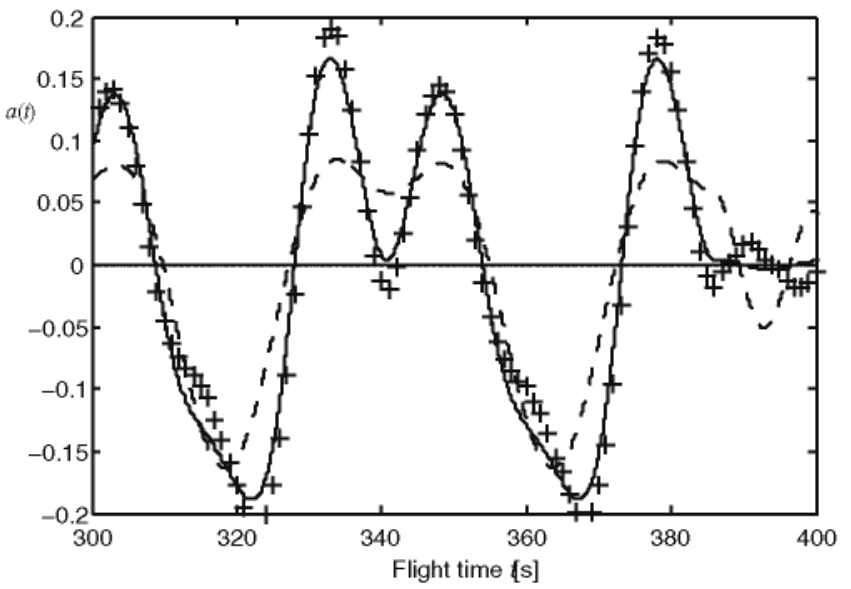

FIG. 9. The amplitude oscillation in a liquid bridge with $\Lambda=2.83$ and $V /\left(\pi L R_{0}{ }^{2}\right)=1$, excited with a sinusoidal Bond number $B(t)=B_{1} \cos \omega t$ $+B_{3} \cos 3 \omega t$ produced by shaking with a crank-shaft mechanism (Ref. 24). Experimental measurements (crosses) are compared with the result obtained via integration of the amplitude equation in two cases: considering all terms in the equation (continuous line) and neglecting the cubic term (dashed line).

present model), which is due to the large-slenderness approximation that is inherent in the Cosserat model, which has not been made in this paper. For the sake of comparison of theory versus experiments, Fig. 9, adapted from Martinez, Perales, and Meseguer, ${ }^{24}$ shows the comparison of the evolution of the measured amplitude evolution of a liquid bridge with $\Lambda=2.83$ and $V /\left(\pi L R_{0}{ }^{2}\right)=1$ excited with a oscillatory Bond number $B(t)=B_{1} \cos \omega t+B_{3} \cos 3 \omega t$ resulting from shaking the bridge with a crank-shaft mechanism. In this figure, the experimental measurements (crosses) are compared with the simulation both considering the full amplitude equation (solid line) and neglecting the cubic term (dashed line). Note that, as it happened in Fig. 8, nonlinearity plays a significant role in the oscillations.

Apart from the quoted works, there are very few more available results. Meseguer et al. ${ }^{45}$ visualized both static configurations close to the instability limit and the breaking of liquid bridges for non cylindrical configurations, restricting to configurations with $\Lambda>\Lambda_{\mathrm{A}}$ (the behavior described in Sec. III). The study focused in comparing the theoretical stability limit with the actual experimental behavior. A short sequence of breakage snapshots was also provided but the time resolution of visualizations does not allow for a sound comparison with the theoretical results in the present work.

\section{CONCLUSIONS}

Two amplitude equations have been derived for the weakly nonlinear dynamics of a liquid bridge when it is close to its stability limit. These two models apply in the two regimes that must be considered, depending on the slenderness. The stability limit considered has been that of minimum volume where two different types of instability may appear, one leading to reflection symmetric deformations (symmetric breakages) with regard to $z$ for $\Lambda_{\mathrm{C}}<\Lambda<\Lambda_{\mathrm{A}}$ and the other leading to reflection antisymmetric deformations 
(antisymmetric breakages) with regard to $z$ for $\Lambda>\Lambda_{\mathrm{A}}$. The different symmetries in the two regimes have dynamic consequences since the amplitude equations are qualitatively different. Namely, in the latter regime the equation is a standard Duffing equation, while in the former case it is a quadratic Duffing-like equation. The amplitude equations also include the effect of the change of volume, disk unequality, and axial gravity, either stationary of slowly varying with time.

The time integration of either of the equations for a given configuration allows for predicting not only the stability or instability of the configuration but also the weakly nonlinear behavior of the configuration. Comparison with the available experimental results (which are concerned with nearly cylindrical configurations) is quite good. Further comparison with experimentally obtained dynamic behavior of truly noncylindrical configurations will be considered elsewhere, when these are available.

${ }^{1}$ J. Meseguer, J. M. Perales, I. Martínez, N. A. Bezdenejnykh, and A. Sanz, "'Hydrostatic instabilities' in floating zone crystal growth process," Curr. Top. Cryst. Growth Res. 5, 27 (1999).

${ }^{2}$ L. Rayleigh, "On the instability of jets," Proc. London Math. Soc. s1-10, 4 (1878).

${ }^{3}$ L. A. Slobozhanin and J. M. Perales, "Stability of liquid bridges between equal disks in an axial gravity field," Phys. Fluids A 5, 1305 (1993).

${ }^{4}$ L. A. Slobozhanin, M. Gómez, and M. J. M. Perales, "Stability of liquid bridges between unequal disks under zero-gravity conditions," Microgravity Sci. Technol. 8, 23 (1995).

${ }^{5}$ L. A. Slobozhanin and J. M. Perales, "Stability of isorotating liquid bridges between equal disks under zero-gravity conditions," Phys. Fluids 8, 2307 (1996).

${ }^{6}$ L. A. Slobozhanin and J. I. D. Alexander, "Stability of an isorotating liquid bridge in an axial gravity field," Phys. Fluids 9, 1880 (1997).

${ }^{7}$ L. A. Slobozhanin and J. I. D. Alexander, "Combined effect of disk inequality and axial gravity on axisymmetric liquid bridge stability," Phys. Fluids 10, 2473 (1998).

${ }^{8}$ A. Laverón-Simavilla and J. M. Perales, "Equilibrium shapes of nonaxisymmetric liquid bridges of arbitrary volume in gravitational fields and their potential energy," Phys. Fluids 7, 1204 (1995).

${ }^{9}$ A. Laverón-Simavilla and E. Checa, "Effect of a lateral gravitational field on the nonaxisymmetric equilibrium shapes of liquid bridges held between eccentric disks and of volumes equal to those of cylinders," Phys. Fluids 9, 817 (1997)

${ }^{10}$ J. M. Vega and J. M. Perales, "Almost cylindrical isorotating liquid bridges for small bond numbers," Eur. Space Agency, [Spec. Publ.] ESA SP ESA SP-193, 247 (1983).

${ }^{11}$ M. Gómez, I. Parra, and J. M. Perales, "Mechanical imperfections effect on the minimum stability limit of liquid bridges," Phys. Fluids 14, 2029 (2002).

${ }^{12}$ J. M. Perales, J. Meseguer, and I. Martínez, "Minimum volume of axisymmetric liquid bridges between unequal disks in an axial microgravity field," J. Cryst. Growth 110, 855 (1991).

${ }^{13}$ N. A. Bezdenejnykh, J. Meseguer, and J. M. Perales, "Experimental analysis of stability limits of capillary liquid bridges," Phys. Fluids A 4, 677 (1992).

${ }^{14}$ N. A. Bezdenejnykh, J. Meseguer, and J. M. Perales, “An experimental analysis of the instability of non-axisymmetric liquid bridges in a gravitational field," Phys. Fluids 11, 3181 (1999).

${ }^{15} \mathrm{~J}$. A. Nicolás, "Frequency response of axisymmetric liquid bridges to an oscillatory microgravity field," Microgravity Sci. Technol. IV, 188 (1991).

${ }^{16}$ D. Langbein, "Oscillations of finite liquid columns," Microgravity Sci. Technol. V, 73 (1992).

${ }^{17}$ Y. Zhang and J. I. D. Alexander, "Sensitivity of liquid bridges subject to axial residual acceleration," Phys. Fluids A 2, 1966 (1990).
${ }^{18}$ J. M. Perales and J. Meseguer, "Theoretical and experimental study of the vibration of axisymmetric viscous liquid bridges," Phys. Fluids A 4, 1110 (1992).

${ }^{19}$ J. A. Nicolás and J. M. Vega, "Linear oscillations of axisymmetric viscous liquid bridges," Z. Angew. Math. Phys. 51, 701 (2000).

${ }^{20} \mathrm{~J}$. M. Montanero, "Theoretical analysis of the vibration of axisymmetric liquid bridges of arbitrary shape," Theor. Comput. Fluid Dyn. 16, 171 (2003).

${ }^{21}$ J. M. Montanero, "Linear dynamics of axisymmetric liquid bridges," Eur. J. Mech. B/Fluids 22, 167 (2003).

${ }^{22}$ A. Gañán and A. Barrero, "Free oscillations of liquid captive drops," Microgravity Sci. Technol. III, 70 (1990).

${ }^{23}$ J. A. Nicolás, D. Rivas, and J. M. Vega, "On the steady streaming flow due to high-frequency vibration in nearly inviscid liquid bridges," J. Fluid Mech. 354, 147 (1998).

${ }^{24}$ I. Martínez, J. M. Perales, and J. Meseguer, "Non-linear response of a liquid bridge to a sinusoidal acceleration under microgravity," Exp. Fluids 37, 775 (2004).

${ }^{25}$ I. Martínez, J. Meseguer, and J. M. Perales, "Wobbling of a liquid column between unequal discs," Adv. Space Res. 36, 26 (2005).

${ }^{26}$ R. M. S. M. Schulkes, "Nonlinear dynamics of liquid columns: A comparative study," Phys. Fluids A 5, 2121 (1993).

${ }^{27} \mathrm{~J}$. Eggers, "Nonlinear dynamics and breakup of free-surface flows," Rev. Mod. Phys. 69, 865 (1997).

${ }^{28} \mathrm{~T}$. Chen and J. Tsamopoulos, "Nonlinear dynamics of capillary liquid bridges: Theory," J. Fluid Mech. 255, 373 (1993).

${ }^{29}$ D. J. Mollot, J. Tsamopoulos, T. Chen, and N. Ashgriz, "Nonlinear dynamics of capillary liquid bridges: Experiments," J. Fluid Mech. 255, 411 (1993).

${ }^{30}$ S. Ahrens, F. Falk, R. Großbach, and D. Langbein, "Experiments on oscillations of small liquid bridges," Microgravity Sci. Technol. VII, 2 (1994).

${ }^{31}$ A. Sanz, "The influence of the outer bath in the dynamics of axisymmetric liquid bridges," J. Fluid Mech. 156, 101 (1985).

${ }^{32}$ M. Higuera, J. A. Nicolás, and J. M. Vega, "Linear oscillations of weakly dissipative axisymmetric liquid bridges," Phys. Fluids 6, 438 (1994).

${ }^{33}$ J. A. Nicolás and J. M. Vega, "Weakly nonlinear oscillations of nearly inviscid axisymmetric liquid bridges," J. Fluid Mech. 328, 95 (1996).

${ }^{34}$ D. Rivas and J. Meseguer, "One-dimensional self-similar solution of the dynamics of axisymmetric slender liquid bridges," J. Fluid Mech. 138, 417 (1984).

${ }^{35}$ F. J. García and A. Castellanos, "One-dimensional models for slender axisymmetric viscous liquid jets," Phys. Fluids 6, 2676 (1994).

${ }^{36}$ F. J. García and A. Castellanos, "One-dimensional models for slender axisymmetric viscous liquid bridges," Phys. Fluids 8, 2837 (1996).

${ }^{37}$ J. M. Montanero and F. J. Acero, "A note on the use of the onedimensional models to describe the linear dynamics of liquid bridges," Eur. J. Mech. B/Fluids 24, 288 (2005).

${ }^{38}$ J. Meseguer, M. A. González, and J. I. D. Alexander, "Dynamic stability of long, axisymmetric liquid bridges," Microgravity Sci. Technol. VII, 246 (1994)

${ }^{39}$ I. Martínez and J. M. Perales, "Liquid bridge stability data," J. Cryst. Growth 78, 369 (1986).

${ }^{40}$ A. H. Nayfeh and D. T. Mook, Nonlinear Oscillations (Wiley, New York, 1979).

${ }^{41}$ J. Guckenheimer and P. Holmes, Nonlinear Oscillations, Dynamical Systems, and Bifurcations of Vector Fields (Springer-Verlag, New York, 1983).

${ }^{42}$ F. C. Moon, Chaotic Vibrations: An Introduction for Applied Scientists and Engineers (Wiley, New York, 1987).

${ }^{43}$ J. M. T. Thompson and H. B. Stewart, Nonlinear Dynamics and Chaos (Wiley, Chichester, 2002).

${ }^{44}$ I. Martínez, I. J. M. Perales, and J. Meseguer, "Stability of long liquid columns (SL-D2-FPM-STACO)," in Scientific Results of the German Spacelab Mission D-2, edited by P. R. Sahm, M. H. Keller, and B. Schiewe, (Wissenschaftliche Projektführung D-2, Koln, 1995).

${ }^{45}$ J. Meseguer, J. L. Espino, J. M. Perales, and A. Laverón-Simavilla, "On the breaking of long, axisymmetric liquid bridges between unequal supporting disks at minimum volume stability limit," Eur. J. Mech. B/Fluids 22, 355 (2003). 